\title{
QUASI-COMPLEMENTS AND CLOSED PROJECTIONS IN REFLEXIVE BANACH SPACES
}

\author{
BY \\ F. J. MURRAY
}

Introduction. Given a closed additive subset $\mathfrak{M}$ of a Banach space, $\mathfrak{B}$, the problem of complementation is fundamental in the study of the structure of the space and also of linear transformations. If we regard $\mathfrak{B}$ and $\mathfrak{M}$ as abelian groups, it corresponds to the problem of finding in $\mathfrak{B}$ a (closed) subgroup, $\mathfrak{N}$, which is isomorphic to $\mathfrak{B} / \mathfrak{M}$ under an "element of" relationship.

More directly, a complement is defined by the statement: A closed additive set $\mathfrak{R}$ is said to be a complement to $\mathfrak{M}$ if every element of $\mathfrak{B}$ has a unique resolution in the form $g+h$ where $g \in \mathfrak{M}$ and $h \in \mathfrak{R}$. The uniqueness of the resolution is equivalent to the statement: $\mathfrak{M} \cdot \mathfrak{N}=(0)$. If we denote the set of elements in the form $g+h, g \in \mathfrak{M}, h \in \mathfrak{R}$, as $\mathfrak{M} \cdot+\cdot \mathfrak{N}$, complementation can be expressed as $\mathfrak{M} \cdot+\cdot \mathfrak{N}=\mathfrak{B}, \mathfrak{M} \cdot \mathfrak{R}=(0)$.

The existence of a complement is equivalent to the statement: There exists a continuous additive transformation $E$ with $E^{2}=E$ and range $\mathfrak{M}$. Such an $E$ is called a continuous or bounded projection. (Cf. [M2]. The square brackets refer to the bibliography at the end of the paper.)

In a previous paper the author has shown that in the separable reflexive Banach space, $\mathfrak{l}_{p}, p>1, p \neq 2$, there exist manifolds $\mathfrak{M}$ which do not have complements ([M2]). A second proof of this has been given in [S].

In an address before the American Mathematical Society on May 2, 1941 ([M4]), the author suggested the study of quasi-complements. $\mathfrak{N}$ is a quasicomplement to $\mathfrak{M}$ if $\mathfrak{M} \cdot \mathfrak{N}=(0)$ and $\mathfrak{M} \cdot+\cdot \mathfrak{N}$ is dense in $\mathfrak{B}$. For quasi-complements, we have closed rather than continuous projections, $E$. (Cf. $\$ 4$ below.)

In the present paper, we prove that for a separable reflexive Banach space $\mathfrak{B}$, with a separable adjoint space, $\mathfrak{B}^{*}$, every closed additive set, $\mathfrak{M}$, has a quasi-complement. For the type of space mentioned, this answers Problem I of the above mentioned address.

In certain ways, this is quite remarkable. For instance, while, in Hilbert space, many essential structure theorems hold for continuous transformations and also for closed transformations (cf. [M3, chap. 9]), the latter are preferred simply because they are more inclusive. But here we have a property which is associated with closed projections rather than the continuous projections. In this case, then, the closed transformations are really more effective than the continuous transformations. These results certainly indicate that it will be very desirable to study the structure of linear transformation relative to closed projections as suggested in [M4].

Presented to the Society, October 30, 1943; received by the editors April 14, 1944. 
However one of the usual weaknesses of the closed transformation theory is indicated in our theorem of $\S 9$, below. A quasi-complement $\mathfrak{N}$ of $\mathfrak{M}$, which is not a complement, has a proper subset, $\mathfrak{R}^{\prime \prime}$, which is also a quasi-complement of $\mathfrak{M}$ and, furthermore, it itself is a proper subset of a quasi-complement $\mathfrak{N}^{\prime}$ to $\mathfrak{M}$. Thus a proper quasi-complement is not only not unique but can be properly expanded or contracted to quasi-complements.

Summary. The main result is given in the theorem of $\S 8$. The cases in which either $\mathfrak{M}$ or its orthogonal complement $\left(^{1}\right) \mathfrak{M}^{P}$ are finite-dimensional are easily disposed of. In the remaining case, we construct a biorthogonal series $\phi_{1}, \phi_{2}, \cdots, \Phi_{1}, \Phi_{2}, \cdots$ with the properties that while the $\phi_{1}, \phi_{2}, \phi_{3}, \cdots$ $\operatorname{span} \mathscr{B}$, the $\phi_{1}, \phi_{3}, \phi_{5}, \cdots$ span $\mathfrak{M}$ and similarly the $\Phi_{1}, \Phi_{2}, \Phi_{3}, \cdots$ span the conjugate space, $\mathfrak{B}^{*}$, and the $\Phi_{2}, \Phi_{4}, \Phi_{6}, \cdots$ span $\mathfrak{M}^{P}$, the orthogonal complement of $\mathfrak{M}$.

Using this series, we define an operation $E^{\prime}$ on the finite linear combinations of the $\phi$ 's by suppressing the $\phi$ 's with even subscripts, that is, $E^{\prime} h$ is defined if $h=\sum_{j=1}^{2 k} a_{j} \phi_{j}$ and $E^{\prime} h=\sum_{s=1}^{k} a_{2 s-1} \phi_{2 s-1}$. Under these circumstances $E^{\prime}$ is a transformation having a dense domain and its adjoint also has a dense domain. This last implies that the closure of $E^{\prime}$ exists. This closure $E$ is the desired projection whose range is $\mathfrak{M}$.

$\$ \$ 1$ and 2 are concerned with notations and certain preliminary notions. These latter include the concepts of orthogonality, relative totality and also the set, $\mathfrak{S}$, of elements minimal relative to a given closed additive $\mathfrak{M}$, a notion discussed in [M4] and in particular Appendix I, pp. 85-87.

In our proof, certain relations between a transformation and its adjoint are fundamental. These relations are based on the graph and were originally given for Hilbert space by von Neumann in [v.N]. They were generalized to $\mathfrak{R}_{p}$ by the author in [M1]. While the generalization to a reflexive Banach space is quite easy, it is given in $\$ 3$ for completeness.

$\$ 4$ gives the relationship between projections and quasi-complements and also certain properties of projections.

The actual construction of the necessary biorthogonal series is given in $\S \S 5-7$. $\$ 5$ is concerned with the "bi-orthogonalizing" process, that is, the equivalent of the Gram-Schmidt process. The form which we use is based on certain adjoint properties of projections and is given in Lemma 15. The series $\phi_{1}, \phi_{2}, \cdots$ stems ultimately from a sequence $\left\{g_{i 0}\right\}$ which is dense in $\mathfrak{M}$ and a sequence $\left\{h_{i 0}\right\}$, dense in $\subseteq$, the set of elements minimal relative to $\mathfrak{M}$. But a certain rectification is necessary. The set $\left\{h_{i}\right\}$ from $\subseteq$ which is used must be linearly independent, modulo $\mathfrak{M}$, and this is accomplished in Lemma 16. Lemma 17 insures that $\subseteq$ be infinite-dimensional, which is essential to the final construction as given in Lemmas 18 and 19.

$\$ 9$ discusses the extension question.

(1) Due to the wartime emergency, $\mathfrak{M}^{P}$ is used instead of $\mathfrak{M}^{\perp}$ for the orthogonal complement. 
1. We deal with a separable Banach space, $\mathscr{B}$, that is, $\mathscr{B}$ is of type $(B)$, additive, normed and complete. (Cf. [B, p. 53].) It will be immaterial in our discussion, as to whether the scalar multiplication of $B$ is by real or complex quantities. Cf. [M1, p. 84] and [BS]. Elements of $B$ will be denoted by $f, g, h$, and $k$. The set of continuous additive functionals on $\mathscr{B}$ constitute again a Banach space $\mathfrak{B}^{*}$. (Cf. for instance, [M3, p. 11].) Elements of $\mathfrak{B}^{*}$ will be denoted by $F, G, H$, and $K$. We assume that $\mathbb{B}^{*}$ is separable also.

$\mathfrak{B}$ is isomorphic with a subset of $\left(\mathfrak{B}^{*}\right)^{*}$, for the equation

$$
F(f)=\eta(F),
$$

for a fixed $f \in \mathscr{B}$, defines a linear functional $\eta$ on the $F \in B^{*}$. This subset is not $\left(\mathfrak{B}^{*}\right)^{*}$ in general. (Cf. [B, pp. 115-116]. This discussion shows that the subset of $\left(\mathfrak{B}^{*}\right)^{*}$ corresponding to $\mathfrak{B}$ does not have a property of $\left(\mathfrak{B}^{*}\right)^{*}$, that is, the property of the lemma of [B, chap. IV, $\$ 3$, p. 57].) We wish however to confine ourselves to the case in which $\mathfrak{B}$ is isomorphic to $\left(\mathfrak{B}^{*}\right)^{*}$. In this case, $\mathfrak{B}$ is called reflexive.

Thus we are concerned with a separable, reflexive, Banach space for which $\mathfrak{B}^{*}$ is also separable. (The last condition may be redundant.) The relationship (1) for all $F$ of $\mathfrak{B}^{*}$ between $f$ and $\eta$ is then an isomorphism and thus we may consistently identify $\eta \in\left(\mathfrak{B}^{*}\right)^{*}$ with the corresponding $f$.

A set $\subseteq$ in $\mathbb{B}$ is said to be additive if the linear combinations of its elements are also in it. If $\mathfrak{S}$ is any set, we denote by $\mathfrak{A}(\mathfrak{S})$ the set of linear combinations of elements of $\mathfrak{S}$. $\mathfrak{S} \subset \mathfrak{A}(\mathfrak{S})$. Additivity may be written as $\mathfrak{S}=\mathfrak{A}(\mathfrak{S})$. It will be convenient to let $\mathfrak{A}$ stand also for an arbitrary additive set.

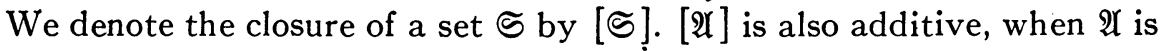
additive. The notations $\mathfrak{M}$ and $\mathfrak{N}$ will be reserved for closed additive sets.

If $\mathfrak{S}$ is an arbitrary subset of $\mathfrak{B}$, we denote by $\mathfrak{S}^{P}$ the set in $\mathfrak{B}^{*}$ of linear functionals $F$ such that $F(g)=0$, for all $g \in \mathfrak{S}$. $\left(\mathfrak{S}^{P}\right)^{P}$ is of course in $\left(\mathfrak{B}^{*}\right)^{*}$. But we should like to identify $\left(\mathfrak{B}^{*}\right)^{*}$ and $\mathscr{B}$ as in the above. Thus we consider $\left(\mathfrak{S}^{P}\right)^{P}$ to be in $\mathfrak{B}$.

Lemma 1. (a) $\varsigma^{P}$ is closed and additive. If $\mathfrak{\Im}_{1} \subset \Im_{2}, \Im_{2}{ }^{P} \subset \Im_{1}{ }^{P}$.

(b) $\mathfrak{S}^{P}=[\mathfrak{S}]^{P}=(\mathfrak{A}(\mathfrak{S}))^{P}=[\mathfrak{A}(\mathfrak{S})]^{P}$.

(c) $\left(\mathfrak{M}^{P}\right)^{P}=\mathfrak{M}$.

Proof. (a) is obvious.

(b) $F(g)=0$ for all $g \in \subseteq$ implies $F(g)=0$ for all $g \in[\Im], \mathfrak{A}(\Im)$, and $[\mathfrak{A}(\mathfrak{S})]$ by the additivity and continuity of $F$. Hence $\mathfrak{S}^{P} \subset \mathfrak{A}(\mathfrak{S})^{P}, \mathfrak{S}^{P} \subset[\subseteq]^{P}$,

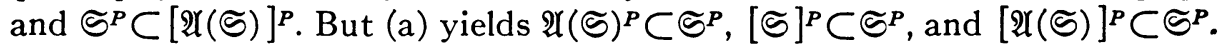
Hence (b) follows.

(c) There is a result of Banach which states that if $h$ is not in $\mathfrak{M}$, there is an $F \in \mathfrak{M}^{P}$ such that $F(h)=1$. (Cf. [B, chap. IV, §3, lemma, p. 57].) Thus $h$ not in $\mathfrak{M}$ implies $h$ not in $\left(\mathfrak{M}^{P}\right)^{P}$. On the other hand $h \in \mathfrak{M}$ clearly implies $h \in\left(\mathfrak{M}^{P}\right)^{P}$ and thus the two sets are identical. 
Note the corollary to (b) and (c).

Corollary. $\left(\Im^{P}\right)^{P}=[\mathfrak{A}(\mathfrak{S})]$.

Proof. Let $\mathfrak{M}=[\mathfrak{A}(\mathfrak{S})]$. Then (b) shows $\mathfrak{S}^{P}=\mathfrak{M}^{P}$ and $(\mathrm{c})$ implies $\left(\mathfrak{S}^{P}\right)^{P}$ $=\left(\mathfrak{M}^{P}\right)^{P}=\mathfrak{M}$.

Suppose now $\mathfrak{S} \subset \mathfrak{B}$ and $\mathfrak{S}^{\prime} \subset \mathfrak{B}^{*}$. $\mathfrak{S}^{\prime}$ will be said to be total relative to $\mathfrak{S}$ if $F(f)=0$ for all $F \in \mathfrak{S}^{\prime}$ and $f \in \mathscr{S}$ imply $f=0$. Identifying $\left(\mathfrak{B}^{*}\right)^{*}$ and $\mathfrak{B}$ we may consistently define: $\subseteq$ is total relative to $\mathfrak{S}^{\prime}$ if $F(f)=0$ for all $f \in \subseteq$ and $F \in \complement^{\prime}$ implies $F=0$.

LEMMA 2. (a) Totality of $\mathfrak{S}^{\prime}$ relative to $\subseteq$ may also be written $\mathfrak{S}^{\prime P} \cdot \mathfrak{S}=(0)$. $\left[\mathfrak{U}\left(\mathfrak{S}^{\prime}\right)\right]$.

(b) Totality relative to $\mathfrak{S}$ of $\mathfrak{S}^{\prime}$ is equivalent to that of $\left[\mathfrak{S}^{\prime}\right], \mathfrak{A}\left(\mathfrak{S}^{\prime}\right)$, or

(c) $\mathfrak{B}^{*}$ is total relative to $\mathfrak{B}$.

(d) If $\mathfrak{S}^{\prime \prime} \supset \mathfrak{S}^{\prime}, \mathfrak{S}^{\prime}$ total relative to $\mathfrak{S}$ implies $\mathfrak{S}^{\prime \prime}$ total relative to $\mathfrak{S}$.

Proof. (a) is obvious. Lemma 1 (b) and Lemma 2(a) yield (b). To show (c), we let $\mathfrak{R}=(0) . \mathfrak{M}^{P}=\mathfrak{B}^{*}$ and Lemma 1 (c) show that $\left(\mathfrak{B}^{*}\right)^{P}=\left(\mathfrak{M}^{P}\right)^{P}=(0)$. Thus $\mathfrak{B}^{* P} \cdot \mathbb{B}=(0)$ and now (a) implies (c). Lemmas 1 (a) and $2(\mathrm{a})$ imply $2(\mathrm{~d})$.

2. We now consider a fixed $\mathfrak{M} \subseteq \mathscr{B}$. ( $\mathfrak{M}$ is of course closed and additive.) Let $\subseteq$ denote the set of elements $h$, minimal relative to $\mathfrak{M}$, that is, for which for every $g \in \mathfrak{M},|h+g| \geqq|h|$. $\subseteq$ is closed since this condition is clearly implied for limit points of $\subseteq$.

Let $f$ be an arbitrary element of $\mathfrak{B}$. Let $C$ be the set of elements $f+g$ for $g \in \mathfrak{M}$. In [M4, Appendix I, p. 86] it is shown that, for every $f, C$ contains an element of $\mathfrak{S}$, that is, $h=f+g, g \in \mathfrak{M}$. We may write this $f=h+(-g), h \in \mathfrak{S}$, $(-g) \in \mathbb{M}$. Thus we have

Lemma 3. Every $f \in \mathfrak{B}$ can be expressed in the form $f=g+h, h \in S, g \in \mathfrak{M}$.

Note that since $|f-g|=|h|$ is a minimum for $g \in \mathfrak{M}, g$ is the element of $\mathfrak{M}$ nearest $f$.

The author has become aware only recently that this and certain other results of the appendix of [M4] had been obtained in [KS].

LEMMA 4. (a) $\subseteq$ is total for $\mathfrak{M}^{P}$.

(b) If $\mathfrak{S}_{0}$ is dense in $\mathfrak{S}_{,} \mathfrak{S}_{0}$ is total for $\mathfrak{M}^{P}$.

Proof. (a) If $F \in \mathfrak{S}^{P} \cdot \mathfrak{M}^{P}$, then we have for every $f \in \mathfrak{B}, F(f)=F(h)+F(g)$ $=0$ (cf. Lemma 3 ). Hence $F=0$. Thus $\mathfrak{S}^{P} \cdot \mathfrak{M}^{P}=(0)$ and Lemma $2(\mathrm{a})$ shows that $\mathfrak{S}$ is total relative to $\mathfrak{M}^{P}$.

(b) If $\mathfrak{S}_{0}$ is dense in $\mathfrak{S},\left[\mathfrak{S}_{0}\right]=\mathfrak{\Im}$ since the latter is closed. Thus Lemmas 2 (b) and $4(\mathrm{a})$ yield $4(\mathrm{~b})$.

We may also consider $\mathfrak{S}^{*}$, the set of elements of $\mathfrak{B}^{*}$ which are minimal relative to $\mathfrak{M}^{P}$. Since, by Lemma $1(\mathrm{c}), \mathfrak{M}=\left(\mathfrak{M}^{P}\right)^{P}$, we have by Lemma 4 : 
Lemma 5. (a) $\mathfrak{S}^{*}$ is total for $\mathfrak{M}$.

(b) If $\mathfrak{S}_{0}^{*}$ is dense in $\mathfrak{S}^{*}$, $\mathfrak{S}_{0}$ is total for $\mathfrak{M}$.

3. In this section certain relations between a transformation $T$ and its adjoint are established. For $\mathfrak{B}=\mathfrak{S}$, these results were given in [v.N] and for $\mathfrak{B}=\Omega_{p}, p>1$, in [M1]. While the generalization offers no difficulty, inasmuch as these results are essential in our discussion they are given completely.

We shall be interested only in transformations on $\mathfrak{B}$, but in this discussion it is more convenient to state results on transformations between Banach spaces. Thus we consider two separable reflexive Banach spaces $\mathfrak{B}_{1}$ and $\mathfrak{B}_{2}$ and transformations between them. In our applications, $\mathfrak{B}_{1}=\mathfrak{B}_{2}=\mathfrak{B}$.

We form $\mathfrak{B}_{1} \oplus \mathfrak{B}_{2}$, the set of ordered pairs $\left\{f_{1}, f_{2}\right\}$ with $f_{1} \in \mathfrak{B}_{1}, f_{2} \in \mathfrak{B}_{2}$, and norm (say)

$$
\left|\left\{f_{1}, f_{2}\right\}\right|=\left(\left|f_{1}\right|^{2}+\left|f_{2}\right|^{2}\right)^{1 / 2} \text {. }
$$

Addition and scalar multiplication are defined as for vectors. Banach has shown that $\left(\mathfrak{B}_{1} \oplus \mathfrak{B}_{2}\right)^{*}$ is isomorphic with $\mathfrak{B}_{1}^{*} \oplus \mathfrak{B}_{2}{ }^{*}$ with respect to the correspondence

$$
\bar{F} \sim\left\{F_{1}, F_{2}\right\} \quad\left(\bar{F} \in\left(\mathfrak{B}_{1} \oplus \mathfrak{B}_{2}\right)^{*}, F_{1} \in \mathfrak{B}_{1}{ }^{*}, F_{2} \in \mathfrak{B}_{2}^{*}\right)
$$

determined by the equation

$$
\bar{F}\left(\left\{f_{1}, f_{2}\right\}\right)=F_{1}\left(f_{1}\right)+F_{2}\left(f_{2}\right) \quad\left(\left\{f_{1}, f_{2}\right\} \in \mathfrak{B}_{1} \oplus \mathfrak{B}_{2}\right)
$$

(cf. [B, chap. IX, $\S 9$, Theorem 14, p. 192]). Clearly this implies that $\mathfrak{B}_{1} \oplus \mathfrak{B}_{2}$ is reflexive. We identify $\left(\mathfrak{B}_{1} \oplus \mathfrak{B}_{2}\right)^{*}$ and $\mathfrak{B}_{1}^{*} \oplus \mathfrak{B}_{2}^{*}$.

If $T$ is a transformation, we term the set of $f^{\prime}$ 's for which $T f$ is defined the domain of $T$ (usually denoted by $D$ ) and the set of $T f^{\prime}$ 's the range of $T(\Re)$. If $T$ is from $\mathfrak{B}_{1}$ to $\mathfrak{B}_{2}$, we call the set of $\{f, T f\}$ for all $f \in \mathfrak{D}$ the graph of $T$. (This set we shall denote by $\mathfrak{T}$.) $\mathfrak{T} \subset \mathfrak{B}_{1} \oplus \mathfrak{B}_{2}$. Similarly if $T$ is from $\mathfrak{B}_{2}$ to $\mathfrak{B}_{1}$ the set of $\{T f, f\}$ constitutes its graph. $T$ will be additive or closed if $\mathfrak{T}$ is respectively additive or closed. The following lemma is obvious.

LEMMA 6. A set $\mathfrak{T} \subset \mathfrak{B}_{1} \oplus \mathfrak{B}_{2}$ is the graph of a transformation $T$ from $\mathfrak{B}_{1}$ to $\mathfrak{B}_{2}$ if and only if $\left\{f, g_{1}\right\} \in \mathfrak{T}$ and $\left\{f, g_{2}\right\} \in \mathfrak{T}$ imply $g_{1}=g_{2}$, that is, when the first element of a pair determines the second element. When $T$ is additive, this condition is equivalent to $\{0, h\} \in \mathfrak{T}$ implies $h=0$. Similarly an additive $\mathfrak{T} \subset \mathfrak{B}_{1} \oplus \mathfrak{B}_{2}$ is the graph of a transformation from $\mathfrak{B}_{2}$ to $\mathfrak{B}_{1}$ if and only if $\{h, 0\} \in \mathfrak{T}$ implies $h=0$.

For an arbitrary transformation, $T$, consider $\mathfrak{A}(\mathfrak{T}),[\mathfrak{T}]$, or $[\mathfrak{A}(\mathfrak{T})]$. If any of these is the graph of a transformation, we call the corresponding transformations, respectively, the additive extension of $T$ (the usual symbol for this is $\tilde{T})$, the closure of $T([T])$, or the closed additive extension $([\widetilde{T}])$.

If $T$ is a transformation from $\mathfrak{B}_{1}$ to $\mathfrak{B}_{2}$ and $\mathfrak{T}^{P} \subset \mathfrak{B}_{1}^{*} \oplus \mathfrak{B}_{2}{ }^{*}$ (cf. the third 
paragraph of this section) is the graph of a transformation $T^{P}$ from $\mathfrak{B}_{2}{ }^{*}$ to $\mathfrak{B}_{1}^{*}$, then we call $T^{P}$ the perpendicular to $T$. When $T^{P}$ exists, $H=T^{P} G$ if and only if for every $\{f, T f\} \in \mathfrak{T}$ we have

$$
0=\{H, G\}(\{f, T f\})=H(f)+G(T f) .
$$

When $T^{P}$ exists $T^{*}=-T^{P}$ is called the "adjoint" of $T . H=T^{*} G$ is equivalent to $-H=T^{P} G$ and, by the above, we have that this is equivalent to the statement that for every $\{f, T f\} \in \mathfrak{T}$

$$
H(f)=G(T f) .
$$

LEMMA 7. If $T^{P}\left(T^{*}\right)$ exists it is a closed additive transformation. $T^{P}\left(T^{*}\right)$ exists if and only if $[\mathfrak{A}(\mathfrak{D})]=\mathfrak{B}_{1}$. (DD is the domain of $T$.)

Proof. The first statement follows from Lemma 1(a). To show the second, we notice that in the following sequence of statements each is equivalent to the next.

(a) $\mathfrak{T}^{P}$ is the graph of a transformation from $\mathfrak{B}_{2}{ }^{*}$ to $\mathfrak{B}_{1}^{*}$.

(b) $\{H, 0\} \in \mathfrak{T}^{P}$ implies $H=0$.

(c) $\mathfrak{D}^{P}=(0)$.

(d) $\mathfrak{B}_{1}=[\mathfrak{H}(\mathfrak{D})]$.

(a) and (b) are equivalent by Lemma 6. By $(\alpha)$ above $\{H, 0\} \in \mathfrak{T}^{P}$ is equivalent to $0=H(f)$ for all $f \in \mathfrak{D}$, that is, to $H \in \mathfrak{D}^{P}$. Thus (b) and (c) are equivalent. Since $(0)^{P}=\mathfrak{B}_{1}$, Lemma 1 and its corollary show that (c) and (d) are equivalent.

Comment. It is customary to say that $\mathfrak{D}$ "spans" $[\mathfrak{A}(\mathfrak{D})]$. When $T$ is additive, $\mathfrak{D}$ is also additive and thus $[\mathfrak{A}(\mathfrak{D})]=\mathfrak{B}_{1}$ becomes simply that $[\mathfrak{D}]=\mathfrak{B}_{1}$ or $\mathfrak{D}$ is dense.

Lemma 8. If $T$ is additive and has domain dense then [ $T$ ] exists if and only if $T^{P}\left(T^{*}\right)$ has domain dense.

Proof. By Lemma 7, $T^{P}$ exists. This lemma also implies that $\left(\mathfrak{T}^{P}\right)^{P}$ is the graph of a transformation if and only if $T^{P}$ has domain dense. But by the corollary to Lemma $1,\left(\mathfrak{T}^{P}\right)^{P}=[\mathfrak{T}]$ for an additive $\mathfrak{T}$. Thus $[\mathfrak{T}]$ is the graph of a transformation if and only if $T^{P}$ has domain dense.

Corollary. When $[T]$ exists, $\left(T^{P}\right)^{P}=[T]=\left(T^{*}\right)^{*}$. Also $[T]^{*}=T^{*}$ and $[T]^{P}=T^{P}$.

Thus if a transformation $T$ is c.a.d.d., that is, closed, additive, and with a dense domain, then $T^{P}$ (and $T^{*}$ ) also has these properties and furthermore the relationship is involutory, that is, $T$ is related to $T^{P}$ as $T^{P}$ is related to $T$.

We add some simple results for c.a.d.d. transformations.

Lemma 9. If $T$ is c.a.d.d., then: 
(a) $T+a \cdot 1$ for any scalar $a$ is also c.a.d.d. $(-T)^{*}=-T^{*},(T+a \cdot 1)^{*}$ $=T^{*}+a \cdot 1,(T+a \cdot 1)^{P}=-\left(-T^{P}+a\right)=T^{P}-a\left(^{2}\right)$.

(b) If $\mathfrak{R}$ is the set of zeros of $T$, that is, the set of f's for which $T f=0$, then $\mathfrak{N}$ is a closed additive set.

(c) If $\Re^{*}$ denotes the range of $T^{P}$ (and of $T^{*}$ ), then $\Re^{* P}=\Re$. Consequently if $\mathfrak{N}^{*}$ is the set of zeros of $T^{P}\left(\right.$ and of $\left.T^{*}\right)$ then $\mathfrak{R}^{P}=\mathfrak{R}^{*}$.

Proof. (a) Suppose $\left(\left\{f_{i}, T f_{i}+a f_{i}\right\}\right)$ is a convergent sequence in the graph of $T+a$. This implies that $\left(\left\{f_{i}, T f_{i}\right\}\right)$ is also convergent and since $\mathfrak{T}$ is closed, the limit is in the form $\{f, T f\}$. Consequently the limit point of the original sequence is in the form $\{f, T f+a f\}$ and hence it is in the graph of $T+a$. This argument shows that the graph of $T+a$ contains all its limit points and hence $T+a$ is closed.

$(-T)^{*}=-T^{*}$ is obvious from $(\beta)$. From $(\beta)$ we also get that $\{H, G\}$ in the graph of $T^{*}$ is equivalent to

$$
H(f)=G(T f)
$$

for all $f \in \mathfrak{D}$. But this is clearly equivalent to

$$
(H+a G)(f)=G(T f+a f),
$$

for all $f \in \mathfrak{D}$. Thus $\{H+a G, G\}$ in the graph of $(T+a)^{*}$ is equivalent to $\{H, G\}$ in the graph of $T^{*}$. Hence $(T+a)^{*}=T^{*}+a$. This yields in turn $-(T+a)^{*}=-\left(T^{*}+a\right)$ or $(T+a)^{P}=-\left(-T^{P}+a\right)=T^{P}-a$.

(b) If $\left(f_{i}\right)$ is a convergent sequence of elements of $\mathfrak{N}$ with limit $f,\left(\left\{f_{i}, 0\right\}\right)$ is a convergent sequence of $\mathfrak{T}$ with limit $\{f, 0\}$. Since $\mathfrak{T}$ is closed and $\left\{f_{i}, 0\right\} \in \mathfrak{T},\{f, 0\} \in \mathfrak{T}$ or $T f=0$. Hence $f \in \mathfrak{N}$. Thus $\mathfrak{R}$ contains all its limit points and is closed. The additivity of $\mathfrak{N}$ is established similarly.

(c) If $H$, is in the range of $T^{P}$ and $f \in \Re$ then there is a $G$ with $\{H, G\} \in \mathfrak{T}^{P}$ and also $\{f, 0\} \in \mathbb{T}$. Hence

$$
0=\{H, G\}(\{f, 0\})=H(f)+G(0)=H(f) .
$$

Since this holds for all $H \in \Re^{*}$, we must have $f \in \Re^{* P}$. Thus $f \in \mathfrak{R}$ implies $f \in \Re^{* P}$ or $\mathfrak{N} \subset \Re^{* P}$. On the other hand if $f \in \Re^{* P},(\gamma)$ holds for all $\{H, G\} \in \mathfrak{T}^{P}$ and hence $\{f, 0\} \in\left(\mathfrak{T}^{P}\right)^{P}=[\mathfrak{T}]=\mathfrak{T}$. But $\{f, 0\} \in \mathfrak{T}$ means $T f=0$ and $f \in \mathfrak{R}$. Thus $f \in \Re^{* P}$ yields $f \in \Re$ and we may conclude $\Re^{* P} \subset \mathfrak{R}$. The two results give $\mathfrak{R}=\mathfrak{R}^{* P}$.

4. We now consider the connection between the notion of quasi-complement and that of projection. These results were given essentially in [M2], but for completeness we give them here in the form which we shall use.

Suppose $\mathfrak{M}$ and $\mathfrak{N}$ are closed additive subsets of $\mathfrak{B}$. Let $\mathfrak{M} \cdot+\cdot \mathfrak{N}$ denote the

(2) In the case of complex scalar multiplication, for instance in Hilbert space, it is customary to define scalar multiplication in $\mathfrak{B}^{*}$ by taking the product of the linear functional and the complex conjugate of $a$ in the usual sense. These formulas then become $(T+a \cdot 1)^{*}$ $=T^{*}+\bar{a} \cdot 1,(T+a)^{P}=-\left(-T^{P}+\bar{\sigma}\right)=T^{P}-\bar{c}$. 
set of $f+g, f \in \mathfrak{M}, g \in \mathfrak{N} . \mathfrak{M}$ and $\mathfrak{N}$ are quasi-complements if $($ a) $[\mathfrak{M} \cdot+\cdot \mathfrak{R}]=\mathfrak{B}$ and (b) $\mathfrak{M} \cdot \mathfrak{R}=(0)$.

A c.a.d.d. transformation $E$ such that $E^{2}=E$ will be called a projection.

LEMMA 10. (a) If $E$ is a projection and $\mathfrak{N}$ is the set of zeros of $E$ and $\mathfrak{M}$ is the range of $E$, then $\mathfrak{M}$ and $\mathfrak{N}$ are closed additive sets and are quasi-complements.

(b) If $\mathfrak{M}$ and $\mathfrak{R}$ are quasi-complements and $E$ is the transformation defined on $\mathfrak{M} \cdot+\cdot \mathfrak{N}$ such that $E(f+g)=f$ for $f \in \mathfrak{M}$ and $g \in \mathfrak{N}$ then $E$ is a projection.

Proof. (a) Since $E$ is c.a.d.d., $\mathfrak{N}$ is closed by Lemma 9 (b).

We must show that $\mathfrak{M}$ is closed. To do this we first prove that $\mathfrak{M}$ is the set $\mathfrak{M}_{1}$ of elements $f$ for which $E f=f$. Clearly $\mathfrak{M}_{1} \subset \mathfrak{M}$. On the other hand, if $f \in \mathbb{M}$, there is a $g$ such that $f=E g$. Since $E^{2}=E, f=E g=E^{2} g=E(E g)=E f$. Hence $f \in \mathfrak{M}_{1}$. Thus $f \in \mathbb{M}$ implies $f \in \mathfrak{M}_{1}$ and we have $\mathfrak{M} \subset \mathfrak{M}_{1}$. The two inclusions imply $\mathfrak{M}_{1}=\mathfrak{M}$.

We can show that $\mathfrak{M}_{1}$ is closed as follows. By Lemma $9(a), E-1$ is closed. Hence by Lemma 9 (b), $\mathfrak{M}_{1}$, the set of zeros of $E-1$, is closed. Thus $\mathfrak{M}$ is also closed.

It only remains to show that $\mathfrak{M}$ and $\mathfrak{N}$ are quasi-complements. Suppose $f$ is in the domain of $E$. Since $E^{2}=E$ we have $E(1-E)=0$ and hence $(1-E) f \in \mathfrak{R}$. Also $E f \in \mathfrak{M}$. Hence $f=E f+(1-E) f$ shows that $f$ is in $\mathfrak{M} \cdot+\cdot \mathfrak{N}$. Thus $\mathfrak{D C M} \cdot+\cdot \mathfrak{R}$. Since $\mathfrak{D}$ is dense, $\mathfrak{M} \cdot+\cdot \mathfrak{N}$ must be dense. On the other hand $f \in \mathfrak{M} \cdot \mathfrak{N}=\mathfrak{M}_{1} \cdot \mathfrak{N}$ implies $E f=f$ and $E f=0$ or $f=0$. This yields $\mathfrak{M} \cdot \mathfrak{N}$ $=(0)$. Thus $\mathfrak{M} \cdot+\cdot \mathfrak{R}$ is dense and $\mathfrak{M} \cdot \mathfrak{R}=(0)$ and $\mathfrak{M}$ and $\mathfrak{R}$ are quasi-complements.

(b) If $h$ is in $\mathfrak{M} \cdot+\cdot \mathfrak{R}$, the resolution $h=f+g$ for $f \in \mathfrak{M}, g \in \mathfrak{N}$ is unique. For if $h=f+g=f_{1}+g_{1}$, with $f_{1} \in \mathfrak{M}$ and $g_{1} \in \mathfrak{N}$ also, then $f-f_{1}=g_{1}-g \in \mathfrak{M} \cdot \mathfrak{R}$ $=(0)$. This implies $f-f_{1}=g_{1}-g=0$. Hence $E h=E(f+g)=f$ is uniquely determined. This uniqueness implies that $E$ is additive and since $\mathfrak{D}=\mathfrak{M} \cdot+\cdot \mathfrak{R}$, $\mathfrak{D}$ is dense.

We now show that $E$ is closed. Let $\left(\left\{f_{i}+g_{i}, f_{i}\right\}\right)$ be a convergent sequence from $E$, the graph of $E$ with limit $\{h, f\} . f_{i} \rightarrow f$ and $f_{i}+g_{i} \rightarrow h$ imply that $g_{i} \rightarrow h-f=g$. Since $\mathfrak{M}$ and $\mathfrak{N}$ are closed, $f \in \mathfrak{M}$ and $g \in \mathfrak{R}$. Thus $h=f+g$ is in $\mathfrak{M} \cdot+\cdot \mathfrak{R}$ and $\{h, f\}$ is in $\mathfrak{E}$. Thus $\mathbb{E}$ cuntains all its limit points and $E$ is closed.

Thus $E$ is c.a.d.d. Since, for $f \in \mathbb{M}, E f=E(f+0)=f$ we also have $E^{2}=E$ and hence the proof of the lemma is complete.

In Lemma $10(a)$, we showed that $\mathfrak{D C M} \cdot+\cdot \mathfrak{N}$. But $\mathfrak{N}$ and $\mathfrak{M}=\mathfrak{M}_{1}$ are in $\mathfrak{D}$ and since $\mathfrak{D}$ is additive, we also have $\mathfrak{M} \cdot+\cdot \mathfrak{N C D}$ and thus we obtain the following corollary.

Corollary. In Lemma $10(\mathrm{a}), \mathfrak{D}=\mathfrak{M} \cdot+\cdot \mathfrak{R}$.

Lemma 11. If $E$ is a projection, then $1-E$ and $E^{*}$ are projections. If $\mathfrak{M}$ and $\mathfrak{N}$ are as in Lemma $10(\mathrm{a})$, then $\mathfrak{M}$ is the set of zeros of $1-E$ and $\mathfrak{R}$ is the range of $1-E$. $E^{*}$ has range $\mathfrak{N}^{P}$ and the set of zeros of $E^{*}$ is $\mathfrak{M}^{P}$. 
Proof. Let us first consider the statements concerning $1-E$. Since, by the corollary to Lemma 10 , the domain of $E$ is $\mathfrak{M} \cdot+\cdot \mathfrak{N}$, this is also the domain of $1-E$. Since $\mathfrak{M}=\mathfrak{M}_{1}$ (cf. the proof of Lemma $10(a)$ ), we have $E(f+g)=f$ for $f \in \mathfrak{M}, g \in \mathfrak{N}$ and $(1-E)(f+g)=g$. Lemma $10(\mathrm{a})$ tells us that $\mathfrak{M}$ and $\mathfrak{N}$ are quasi-complements. Thus for the quasi-complements, $\mathfrak{M}$ and $\mathfrak{R}, 1-E$ is the transformation defined on $\mathfrak{M} \cdot+\cdot \mathfrak{N}$ such that $(1-E)(f+g)=g$. Hence Lemma 10 (b) shows that $1-E$ is a projection and the other statements on $1-E$ are clear.

We next consider $E^{*}$. $E^{*}$ is c.a.d.d., since $E$ is c.a.d.d. It follows from Lemmas 9 (c) and 1 that $\Re^{*}$, the range of $E^{*}$, is included in $\mathfrak{R}^{P}$. (For $\Re^{* P}=\mathfrak{R}$ implies $\left[\mathfrak{A}\left(\mathfrak{R}^{*}\right)\right]=\mathfrak{R}^{P}$.) On the other hand, if $F \in \mathfrak{R}^{P}$, we have for all $h=f+g$, $f \in \mathfrak{M}, g \in \mathfrak{N}$,

$$
F(h)=F(f+g)=F(f)+F(g)=F(f)=F(E h) .
$$

Thus $(\beta)$ of $\S 3$ yields $E^{*} F$ exists and equals $F$. Hence $\Re^{*}$ also includes $\mathfrak{R}^{P}$ and we may conclude $\Re^{*}=\mathfrak{R}^{P}$. Since $E^{*} F=F$ for $F \in \Re^{*}$, we have $E^{*}\left(E^{*} H\right)$ exists and equals $E^{*} H$ for every $H \in \mathfrak{B}^{*}$ for which $E^{*} H$ is defined. Hence $E^{* 2}=E^{*}$.

Thus we have shown that $E^{*}$ is c.a.d.d. with $\left(E^{*}\right)^{2}=E^{*}$ and $\Re^{*}=\mathfrak{R}^{P}$. Lemma 9 (c) also tells us that $\mathfrak{N}^{*}=\mathfrak{M}^{P}$ and thus the lemma is established.

CoRollary. $\mathfrak{M}$ and $\mathfrak{N}$ are quasi-complements if and only if $\mathfrak{M}^{P}$ and $\mathfrak{N}^{P}$ are quasi-complements.

Proof. If $\mathfrak{M}$ and $\mathfrak{N}$ are quasi-complements, we consider the $E$ of Lemma 10 (b). For this we have by Lemma 11 that $E^{*}$ is a projection with $\mathfrak{M}^{P}$ as set of zeros and $\mathfrak{N}^{P}$ as range. Hence Lemma $10\left(\right.$ a) shows that $\mathfrak{N}^{P}$ and $\mathfrak{N}^{P}$ are quasi-complements.

If on the other hand, $\mathfrak{M}^{P}$ and $\mathfrak{N}^{P}$ are quasi-complements, the above argument shows that $\mathfrak{M}=\left(\mathfrak{M}^{P}\right)^{P}$ and $\mathfrak{R}=\left(\mathfrak{N}^{P}\right)^{P}$ are quasi-complements.

5. We next consider the notion of a biorthogonal series. (Cf. [B, p. 106].) If $\phi_{1}, \phi_{2}, \cdots$ is a (finite or infinite) sequence of elements of $\mathscr{B}$ and $\Phi_{1}, \Phi_{2}, \ldots$ a sequence of $\mathfrak{B}^{*}$ containing as many elements as the first sequence, such that

$$
\Phi_{i}\left(\phi_{j}\right)=\delta_{i, j}
$$

the pair of sequences constitutes a biorthogonal series. This notion is svmmetric, relative to $\mathfrak{B}$ or $\mathfrak{B}^{*}$.

LEMma 12. Let $\phi_{1}, \phi_{2}, \cdots, \Phi_{1}, \Phi_{2}, \cdots$ constitute a biorthogonal series. If an element $h$ of $\mathfrak{B}$ can be expressed as a finite sum

$$
h=\sum_{i=1}^{n} a_{i} \phi_{i}
$$

then the $a_{i}$ 's are uniquely determined and $a_{i}=\Phi_{i}(h)$. 
Proof. Apply $\Phi_{i}$ to both sides of the equation.

LEMMA 13. Suppose $\phi_{1}, \cdots, \phi_{n}, \Phi_{1}, \cdots, \Phi_{n}$ are a given finite biorthogonal series. Let $h \in \mathfrak{B}$ and $H \in \mathfrak{B}^{*}$ be given and let $h^{\prime}=h-\sum_{i=1}^{n} \Phi_{i}(h) \phi_{i}$ and $H^{\prime}=H-\sum_{i=1}^{n} H\left(\phi_{i}\right) \Phi_{i}$. Suppose now that $H^{\prime}\left(h^{\prime}\right) \neq 0$. Then we can enlarge the biorthogonal series $\phi_{1}, \cdots, \phi_{n}, \Phi_{1}, \cdots, \Phi_{n}$, by adding a $\phi_{n+1}, \Phi_{n+1}$, so that the result is a biorthogonal series and $h$ is a linear combination of $\phi_{1}, \cdots, \phi_{n+1}$, and $H$ is a linear combination of the $\Phi_{1}, \cdots, \Phi_{n+1}$.

Proof. It is easy to show that $\Phi_{i}\left(h^{\prime}\right)=0$ for $i=1, \cdots, n$ and $H^{\prime}\left(\phi_{i}\right)=0$ for $i=1, \cdots, n$. Now choose constants $c$ and $C$ so that $c \cdot C H^{\prime}\left(h^{\prime}\right)=1$. Let $\phi_{n+1}=c h^{\prime}, \Phi_{n+1}=C H^{\prime}$. Then $\phi_{1}, \cdots, \phi_{n+1}, \Phi_{1}, \cdots, \Phi_{n+1}$ is the desired extension. For this is readily seen to constitute a biorthogonal series and since $c \neq 0$ and $C \neq 0, h$ and $H$ are linear combinations of, respectively, $\phi_{1}, \cdots, \phi_{n+1}$ and $\Phi_{1}, \cdots, \Phi_{n+1}$.

Lemma 14. Let $\phi_{1}, \cdots, \phi_{n}, \Phi_{1}, \cdots, \Phi_{n}$ be a (finite) biorthogonal series. Then

$$
E h=\sum_{i=1}^{n} \Phi_{i}(h) \phi_{i}
$$

defines a continuous additive transformation with $E^{2}=E$. $E$ is a projection with range $\mathfrak{A}\left(\phi_{1}, \cdots, \phi_{n}\right)$ and

$$
E^{*} F=\sum_{i=1}^{n} F\left(\phi_{i}\right) \Phi_{i}
$$

Proof. It is clear that for every $h$ in $\mathscr{B}$

$$
|E h| \leqq \sum_{i=1}^{n}\left|\Phi_{i}(h) \phi_{i}\right| \leqq \sum_{i=1}^{n}\left|\Phi_{i}(h)\right| \cdot\left|\phi_{i}\right| \leqq\left(\sum_{i=1}^{n}\left|\Phi_{i}\right| \cdot\left|\phi_{i}\right|\right) \cdot|h|
$$

Thus $E$ is bounded and since it is additive, it is continuous. This of course implies that $E$ is c.a.d.d. Now if $h$ is in the form $\sum_{i=1}^{n} a_{i} \phi_{i}, \Phi_{i}(h)=a_{i}$ by Lemma 12 and hence $E h=\sum_{i-1}^{n} a_{i} \phi_{i}=h$. Thus $E h=h$ for all $h$ in the range of $E$ and this implies $E^{2}=E$. Hence $E$ is a projection. Clearly the range of $E$ is $\mathfrak{A}\left(\phi_{1}, \cdots, \phi_{n}\right)$.

Now if, for an $F \in B^{*}$, we define $G=\sum_{i=1}^{n} F\left(\phi_{i}\right) \Phi_{i}$, we have for every $h \in \mathfrak{B}$,

$$
F(E h)=\sum_{i=1}^{n} \Phi_{i}(h) F\left(\phi_{i}\right)=G(h) .
$$

Hence $E^{*} F$ is defined and equals $G$. This proves our statement on $E^{*}$.

LeMma 15. If in the hypothesis of Lemma 13 we have either $H^{\prime}(h) \neq 0$ or $H\left(h^{\prime}\right) \neq 0$ instead of $H^{\prime}\left(h^{\prime}\right) \neq 0$, the conclusion is still valid. 
Proof. This lemma follows from $H^{\prime}(h)=H^{\prime}\left(h^{\prime}\right)=H\left(h^{\prime}\right)$ which we now prove. We define $E$ by the equation $E f=\sum_{i=1}^{n} \Phi_{i}(f) \phi_{i}$. Lemma 14 shows that $E$ is a projection and $h^{\prime}=(1-E) h, H^{\prime}=\left(1-E^{*}\right) H$. Lemma 9 implies that $1-E^{*}=(1-E)^{*}$ and thus $H^{\prime}=(1-E)^{*} H$. Lemma 11 yields that $1-E$ and $(1-E)^{*}$ are projections. Using these results we obtain

$$
\begin{aligned}
H^{\prime}\left(h^{\prime}\right) & =H^{\prime}((1-E) h)=(1-E)^{*} H^{\prime}(h)=(1-E)^{*}{ }^{2} H(h) \\
& =(1-E)^{*} H(h)=H^{\prime}(h), \\
H^{\prime}\left(h^{\prime}\right) & =(1-E)^{*} H\left(h^{\prime}\right)=H\left((1-E) h^{\prime}\right)=H\left((1-E)^{2} h\right) \\
& =H((1-E) h)=H\left(h^{\prime}\right)
\end{aligned}
$$

and this establishes the lemma.

6. We now suppose that $\mathfrak{M}$ is fixed and we begin the construction that will result in a projection with range $\mathfrak{M}$. We recall from $\S 2$ that $\subseteq$ is the set of elements of $\mathfrak{B}$ minimal relative to $\mathfrak{M}$ and $\mathfrak{S}^{*}$ is the set of elements of $\mathfrak{B}^{*}$ minimal relative to $\mathfrak{M}^{P}$.

LEMmA 16. There exist sequences (1) $\left\{\dot{g}_{i}\right\} \subset \mathfrak{M},(2)\left\{G_{i}\right\} \subset S^{*},(3)\left\{h_{i}\right\} \subset \subseteq$, and (4) $\left\{H_{i}\right\} \subset \mathfrak{M}^{P}$ such that:

(a) $\left[\left\{g_{i}\right\}\right]=\mathfrak{M}$.

(b) $\left[\mathfrak{A}\left(\left\{G_{i}\right\},\left\{H_{i}\right\}\right)\right]=\mathscr{B}^{*}$.

(c) $\left[\mathscr{A}\left(\left\{g_{i}\right\},\left\{h_{i}\right\}\right)\right]=\mathfrak{B}$.

(d) $\left[\left\{H_{i}\right\}\right]=\mathfrak{M}^{P}$.

(e) $\sum_{i=1}^{n} a_{i} G_{i} \in \mathfrak{M}^{P}$ implies $a_{i}=0$.

(f) $\sum_{i=1}^{n} a_{i} h_{i} \in \mathbb{M}$ implies $a_{i}=0$.

(g) $\left\{G_{i}\right\}$ is total relative to $\mathfrak{M}$.

(h) $\left\{h_{i}\right\}$ is total relative to $\mathfrak{M}^{P}$.

Proof. Since $\mathscr{B}$ is separable, every subset contains a denumerable or finite set which is dense in it. (Cf. [M3, p. 16, Lemma 1].) This also holds for $\mathfrak{B}^{*}$. Now let $\left\{g_{i 0}\right\}$ be a dense denumerable subset of $\mathfrak{M},\left\{G_{i 0}\right\}$ one for $\mathfrak{S}^{*},\left\{h_{i 0}\right\}$ for $\mathfrak{S}$, and $\left\{H_{i 0}\right\}$ for $\mathfrak{M}^{P}$. From Lemma 3 , we see that $\left\{h_{i 0}\right\} \cdot+\cdot\left\{g_{i 0}\right\}$ is dense in $\mathfrak{B}$ and $\left\{G_{i 0}\right\} \cdot+\cdot\left\{H_{i 0}\right\}$ is dense in $\mathfrak{B}^{*}$. From Lemma $5(\mathrm{~b})$ we get that $\left\{G_{i 0}\right\}$ is total for $\mathfrak{M}$, and from Lemma $4(\mathrm{~b})$ we get that $\left\{h_{i 0}\right\}$ is total for $\mathfrak{M}^{P}$.

We now consider the $G_{i 0}$ successively and if, for any set of $a_{i}$ 's, $G_{n 0}-\sum_{i=1}^{n-1} a_{i} G_{i 0}=K_{n 0} \in \mathfrak{M}^{P}$, we discard $G_{n 0}$ from the $G_{i 0}$ set and add $K_{n 0}$ to the $H_{i 0}$ set. Renumbering if necessary, we obtain new sequences $\left\{G_{i}\right\}$, $\left\{H_{i}\right\}$, for which one still has $\left\{G_{i}\right\} \subset \mathfrak{S}^{*},\left\{H_{i}\right\} \subset \mathfrak{M}^{P}$ but which will also satisfy (b), (d), (e) and (g) as we shall now prove.

(e) is evident from the casting out process. Since $\mathfrak{M} \supset\left\{H_{i}\right\} \supset\left\{H_{i 0}\right\}$ and the latter is dense in $\mathfrak{M}$, (d) is also clear. Now from the casting out process, we see that $\mathfrak{A}\left(\left\{H_{i}\right\},\left\{G_{i}\right\}\right) \supset\left\{G_{i 0}\right\}$. Also $\mathfrak{A}\left(\left\{H_{i}\right\},\left\{G_{i}\right\}\right) \supset\left\{H_{i}\right\} \supset\left\{H_{i 0}\right\}$. Hence $\mathfrak{A}\left(\left\{H_{i}\right\},\left\{G_{i}\right\}\right) \supset\left\{H_{i 0}\right\} \cdot+\cdot\left\{G_{i 0}\right\}$ and thus $\mathfrak{B}^{*} \supset\left[\mathfrak{A}\left(\left\{H_{i}\right\},\left\{G_{i}\right\}\right)\right]$ $\supset\left[\left\{H_{i 0}\right\} \cdot+\cdot\left\{G_{i 0}\right\}\right]=\mathfrak{B}^{*}$. This is (b). 
Only $(\mathrm{g})$ remains. We know that the sequence $\left\{G_{i 0}\right\}$ is total relative to $\mathfrak{M}$. This means that to every $f \in \mathbb{M}$, there is a $G_{n 0}$ such that $G_{n(0)}(f) \neq 0$. Let us consider such an $f \in \mathfrak{M}$ and let $n$ be the least subscript such that $G_{n 0}(f) \neq 0$. This $G_{n 0}$ can not be discarded. For if $G_{n 0}$ is to be discarded, we have $G_{n 0}=\sum_{i=1}^{n-1} a_{i} G_{i 0}+K_{n}$ for a $K_{n} \in \mathfrak{M}^{P}$. By the definition of $n, G_{i 0}(f)=0$ for $i<n$ and since $K_{n} \in \mathfrak{M}^{P}, f \in \mathfrak{M}$, we have $K_{n}(f)=0$. Consequently, we would have $G_{n 0}(f)=\sum_{i=1}^{n=1} a_{i} G_{i 0}(f)+K_{n}(f)=0$ contrary to hypothesis. Hence $G_{n 0}$ can not be discarded and is among the $G_{i}$. Thus we have shown that to every $f \in \mathfrak{M}$, there is a $G_{n 0} \in\left\{G_{i}\right\}$ for which $G_{i}(f) \neq 0$. Thus the new set $\left\{G_{i}\right\}$ is total for $\mathfrak{M}$.

We now rectify the $\left\{h_{i 0}\right\} \subset \subseteq \subseteq$ and $\left\{g_{i 0}\right\} \subset \mathbb{M}$ by discarding those $h_{n 0}$ for which $h_{n 0}=\sum_{i=1}^{n-1} a_{i} h_{i}+k_{n}$ for a $k_{n} \in \mathfrak{M}$ and inserting the $k_{n}$ in the $\left\{g_{i 0}\right\}$ sequence. The resulting sequences $\left\{h_{i}\right\} \subset \subseteq$ and $\left\{g_{i}\right\} \subset \mathbb{M}$ will satisfy (a), (c), $(f)$, and (h) as a proof similar to the above will show.

It will be convenient to consider separately the case in which both $\mathfrak{M}$ and $\mathfrak{M}^{P}$ are infinite-dimensional. The following lemma is important in this case.

LEMMA 17. If a set $\left\{G_{i}\right\}$ is total relative to $\mathfrak{M}$ and $\left\{G_{i}\right\}$ spans only a finitedimensional linear set, then $\mathfrak{M}$ is finite-dimensional.

Proof. Suppose $G_{1}, \cdots, G_{n}$ is a maximal linearly independent set among the $G_{i}$. (The subscripts may have been renumbered.) Since $\mathscr{\Re}\left(G_{1}, \cdots, G_{n}\right)$ $\supset\left\{G_{i}\right\}, \mathfrak{A}\left(G_{1}, \cdots, G_{n}\right)$ is total relative to $\mathfrak{M}$ also by Lemma $2(\mathrm{~d})$. Lemma 2(b) then shows that $G_{1}, \cdots, G_{n}$ is also total relative to $\mathfrak{M}$.

Next we notice that if $G_{m}$ is such that there is a $K_{m} \in \mathfrak{M}^{P}$, such that $G_{m}-\sum_{i=1}^{m-1} a_{i} G_{i}=K_{m}$ for a set of constants $a_{i}$, then we may drop all these $G_{m}$ 's out of the sequence and the result will still be total. The argument is the same as for the proof of $(\mathrm{g})$ of the preceding lemma. Thus we may suppose that if $\sum_{i=1}^{n} a_{i} G_{i} \in \mathfrak{M}^{P}$, then $a_{i}=0$, that is, our $G_{1}, \cdots, G_{n}$ satisfy (e) of the preceding lemma.

We now construct a biorthogonal series, $\phi_{1}, \cdots, \phi_{n}, \Phi_{1}, \cdots, \Phi_{n}$ such that $\phi_{i} \in \mathfrak{M}$ and $\mathfrak{A}\left(\Phi_{1}, \cdots, \Phi_{n}\right)=\mathfrak{H}\left(G_{1}, \cdots, G_{n}\right)$. Suppose now that $\phi_{1}, \cdots, \phi_{k}, \Phi_{1}, \cdots, \Phi_{k}$ have been constructed ( $k$ may be 0$)$ with $\phi_{1}, \cdots, \phi_{k} \in \mathfrak{M}$, and $\mathfrak{A}\left(\Phi_{1}, \cdots, \Phi_{k}\right)=\mathfrak{A}\left(G_{1}, \cdots, G_{k}\right)$. Let $G_{k+1}^{\prime}=G_{k+1}$ $-\sum_{i=1}^{k} G_{k+1}\left(\phi_{i}\right) \Phi_{i}$. Now $G_{k+1}^{\prime}$ is not in $\mathfrak{M}^{P}$, by our hypotheses. Hence there is an $h \in \mathbb{M}$, such that $G_{k+1}^{\prime}(h) \neq 0$. Thus we may apply Lemma 15 to obtain $\phi_{k+1}=c h^{\prime}$ and $\Phi_{k+1}=C G_{k+1}^{\prime}$.

Now $\phi_{k+1}=c h^{\prime}=c\left(h-\sum_{i=1}^{k} \Phi_{i}(h) \phi_{i}\right) \in \mathfrak{M}$ since $h$ and $\phi_{1}, \cdots, \phi_{k}$ are in $\mathfrak{M}$. Since $\mathfrak{A}\left(\Phi_{1}, \cdots, \Phi_{k}\right)=\mathfrak{A}\left(G_{1}, \cdots, G_{k}\right)$ and $C \neq 0$, simple linearity considerations will show that $\mathfrak{A}\left(\Phi_{1}, \cdots, \Phi_{k+1}\right)=\mathfrak{A}\left(G_{1}, \cdots, G_{k+1}\right)$.

Our induction will carry on for $k=0, \cdots, n-1$ and thus we have the desired biorthogonal series.

Since $\mathfrak{A}\left(\Phi_{1}, \cdots, \Phi_{n}\right)=\mathfrak{A}\left(G_{1}, \cdots, G_{n}\right)$ is total for $\mathfrak{M}, \Phi_{1}, \cdots, \Phi_{n}$ is total for $\mathfrak{M}$ by Lemma $2(\mathrm{~b})$. Let $h^{\prime}=h-\sum_{i=1}^{n} \Phi_{i}(h) \phi_{i}$ for any $h \in \mathfrak{M}$. Now 
$h^{\prime} \in \mathfrak{M}$ since $h$ and $\phi_{1}, \cdots, \phi_{n} \in \mathfrak{M}$. Also $\Phi_{i}\left(h^{\prime}\right)=0$ for $i=1, \cdots, n$, and thus the totality of $\Phi_{1}, \cdots, \Phi_{n}$ yields that $h^{\prime}=0$. Thus $h \in \mathfrak{A}\left(\phi_{1}, \cdots, \phi_{n}\right)$ and $\mathfrak{M}$ is finite-dimensional.

Thus when we assume that neither $\mathfrak{M}$ nor $\mathfrak{M}^{P}$ are finite-dimensional, Lemma 17 and Lemma $16(\mathrm{~g})$ and $(\mathrm{h})$ show that both the $\left\{G_{i}\right\}$ and $\left\{h_{i}\right\}$ span infinite-dimensional linear sets.

We may also point out that we may substitute for our finite-dimensional condition in our hypothesis that the $\left\{G_{i}\right\}$ be finite-dimensional modulo $\mathfrak{M}^{P}$. The $G_{1}, \ldots, G_{n}$ in the first paragraph may be chosen as linearly independent modulo $\mathfrak{M}^{P}$ without loss of totality or they may be chosen simply as linearly independent as in the above proof and then the casting out process will reduce them to a finite set.

CoRollary. If a set $\left\{G_{i}\right\}$ is total relative to $\mathfrak{M}$ and the $\left\{G_{i}\right\}$ are finite-dimensional modulo $\mathfrak{M}^{P}$, then $\mathfrak{M}$ is finite-dimensional.

7. In this section, we pass from the previously constructed elements to a biorthogonal series. In the essential case, we have the following lemma.

LEMMA 18. If both $\mathfrak{M}$ and $\mathfrak{M}^{P}$ are infinite-dimensional there exists a biorthogonal series $\phi_{1}, \phi_{2}, \ldots, \Phi_{1}, \Phi_{2}, \ldots$ such that:

(a) $\phi_{2 k+1} \in \mathbb{M}$ for $k=0,1,2, \cdots$.

(b) $\Phi_{2 k} \in \mathfrak{M}^{P}$ for $k=1,2, \cdots$.

(c) Each $g_{i} \in \mathcal{A}\left(\left\{\phi_{1}, \phi_{3}, \phi_{5}, \cdots\right\}\right)$.

(d) Each $H_{i} \in \mathfrak{A}\left(\left\{\Phi_{2}, \Phi_{4}, \Phi_{6}, \cdots\right\}\right)$.

(e) Each $h_{i} \in \mathfrak{R}\left(\left\{\phi_{i}\right\}\right)$.

(f) Each $G_{i} \in \mathscr{R}\left(\left\{\Phi_{j}\right\}\right)$.

Construction. We construct the $\phi_{1}, \phi_{2}, \cdots, \Phi_{1}, \Phi_{2}, \cdots$ by four varying steps which are taken in rotation.

Step I. This is for $\phi_{4 k+1}, \Phi_{4 k+1}, k=0,1,2, \cdots$. We suppose we have $\phi_{1}, \cdots, \phi_{4 k}, \Phi_{1}, \cdots, \Phi_{4 k}$ already defined with the requisite properties (a) and (b). We form, for every $i$,

$$
g_{i}^{\prime}=g_{i}-\sum_{j=1}^{4 k} \Phi_{j}\left(g_{i}\right) \phi_{j}
$$

Since $g_{i} \in \mathfrak{M}$ and $\Phi_{2 s} \in \mathfrak{M}^{P}$ for $s=1, \cdots, 2 k$ by (b), we have $\Phi_{2 s}\left(g_{i}\right)=0$ and

$$
g_{i}^{\prime}=g_{i}-\sum_{i=0}^{2 k-1} \Phi_{2 s+1}\left(g_{i}\right) \phi_{2 s+1} \text {. }
$$

Since $g_{i} \in \mathfrak{M}$ by Lemma 16, (a) for $s=0,1, \cdots, 2 k-1$ implies that $g^{\prime}{ }_{i} \in \mathfrak{M}$. Since $\mathfrak{M}$ is not finite-dimensional, (a) of Lemma 16 implies that not every $g^{\prime}{ }_{i}$ is zero. Let $i^{\prime}$ be the least $i$ such that $g^{\prime}{ }_{i} \neq 0$.

Thus $g_{i^{\prime}}^{\prime} \neq 0$ and $g^{\prime}{ }^{\prime} \in \mathbb{M}$ while $(\mathrm{g})$ of Lemma 16 yields that the set $\left\{G_{i}\right\}$ 
is total for $\mathfrak{M}$. Thus there is a $G_{i^{\prime}}$ such that $G_{j^{\prime}}\left(g^{\prime}{ }^{\prime}\right) \neq 0$.

We now apply Lemma 15 to the biorthogonal series $\phi_{1}, \cdots, \phi_{4 k}$, $\Phi_{1}, \cdots, \Phi_{4 k}$ and $g_{i^{\prime}}, G_{j^{\prime}}$. We can therefore augment the series with a $\phi_{4 k+1}$, $\Phi_{4 k+1}$ such that $g_{i^{\prime}}$ and $G_{j^{\prime}}$ are linear combinations of the $\phi_{1}, \cdots, \phi_{4 k+1}$, $\Phi_{1}, \cdots, \Phi_{4 k+1}$, respectively.

We note (1) $\phi_{4 k+1}=c g^{\prime}{ }_{i} \in \mathfrak{M}$, (2) $g_{i}$ is a linear combination of the $\phi_{1}, \phi_{3}, \cdots, \phi_{4 k+1}$ for $i \leqq i^{\prime}$, and every time this step is taken the corresponding $i^{\prime}$ is at least one larger. This implies $i^{\prime} \geqq k$.

Step II. This is for the $\phi_{4 k+2}, \Phi_{4 k+2}, k=0,1, \ldots$. We suppose that we have $\phi_{1}, \cdots, \phi_{4 k+1}, \Phi_{1}, \cdots, \Phi_{4 k+1}$ already defined with the requisite properties (a) and (b). We define for every $i$,

$$
H^{\prime}{ }_{i}=H_{i}-\sum_{j=1}^{4 k+1} H_{i}\left(\phi_{j}\right) \Phi_{j}
$$

Since $H_{i} \in \mathfrak{M}^{P}$ and $\phi_{2 s+1} \in \mathfrak{M}$ for $s=0, \cdots, 2 k$, we have

$$
H^{\prime}{ }_{i}=H_{i}-\sum_{i=0}^{2 k} H_{i}\left(\phi_{2 s}\right) \Phi_{2 a}
$$

Thus (b) yields that $H^{\prime}{ }_{i} \in \mathfrak{M}^{P}$. Let $i^{\prime}$ be the least $i$ such that $H_{i^{\prime}} \neq 0$. Lemma $16(\mathrm{~h})$ and the above results show that there is an $h_{j^{\prime}}$ such that $H^{\prime}{ }^{\prime}{ }^{\prime}\left(h_{j^{\prime}}\right) \neq 0$. We may again apply Lemma 15 to obtain a $\phi_{2 k+2}=c h^{\prime}{ }_{j^{\prime}}$ and $\Phi_{2 k+2}=C H^{\prime}{ }^{\prime}{ }^{\prime}$.

We again note (1) $\Phi_{4 k+2} \in \mathfrak{M}^{P},(2)$ there is an $i^{\prime} \geqq k$ such that $H_{1}, \cdots, H_{i^{\prime}}$ are all in $\mathfrak{A}\left(\Phi_{1}, \cdots, \Phi_{4 k+2}\right)$.

Step III. This is to construct $\phi_{4 k+3}, \Phi_{4 k+3}$, assuming (a) and (b) for $\phi_{1}, \cdots, \phi_{4 k+2}, \Phi_{1}, \cdots, \Phi_{4 k+2}$.

Form, for all $i$,

$$
G_{i}^{\prime}=G_{i}-\sum_{j=1}^{4 k+2} G_{i}\left(\phi_{j}\right) \Phi_{j}
$$

Now not all the $G_{i}^{\prime}$ 's are zero, because if they were, the $\left\{G_{i}\right\}$ would be contained in a finite-dimensional set, which contradicts the result after Lemma 17 that the $\left\{G_{i}\right\}$ span an infinite-dimensional manifold. Let $i$ ' be the least $i$ such that $G^{\prime}{ }_{i} \neq 0$.

We next show that $G^{\prime}{ }_{i}$ is not in $\mathfrak{M}^{P}$. For each $\Phi_{j}$ for $j \leqq 4 k+2$ is a linear combination of certain $H$ 's and $G$ 's. The $G$ 's which appear are those, $G_{a_{1}}, \cdots, G_{s_{t}}$, which have been used in steps $4 s+1, s \leqq k$, or $4 s+3, s<k$, and hence are linear combinations of the $\Phi_{1}, \cdots, \Phi_{2 k+2}$, Consequently the result of priming these $G$ 's is zero, while $G_{i^{\prime}}^{\prime} \neq 0$. Hence $G_{i^{\prime}} \neq G_{s_{j}}$ for $j=1, \cdots, t$. Thus when we eliminate $\Phi_{1}, \cdots, \Phi_{4 k+2}$, in the expression for $G^{\prime}{ }^{\prime}$, we get

$$
G^{\prime}{ }^{\prime}{ }^{\prime}=G_{i^{\prime}}+\sum_{j=1}^{t} a_{j} G_{s_{j}}+H
$$


where no $G_{s^{j}}=G_{i^{\prime}}$ and $H \in \mathfrak{M P}^{P}$. Thus $G^{\prime}{ }_{i^{\prime}} \in \mathfrak{M P}^{P}$ leads to

$$
G_{i}+\sum_{j=1}^{t} a_{j} G_{s_{j}} \in \mathfrak{M}^{P}
$$

which contradicts (e) of Lemma 16 . It follows that $G^{\prime}{ }_{i}$, is not in $\mathfrak{M}^{P}$ and that there is a $j$ such that $G^{\prime}{ }_{i}\left(g_{j}\right) \neq 0$. Let $j^{\prime}$ be the least such $j$.

We now apply Lemma 15 to $G_{i^{\prime}}, g_{j^{\prime}}$ and obtain $\phi_{4 k+3}=c g^{\prime}{ }_{i^{\prime}}, \Phi_{4 k+3}=C G^{\prime}{ }_{i^{\prime}}$.

Again we may remark (1) $\phi_{4 k+3} \in \mathfrak{M}[(a)$ and (b) for subscripts less than $4 k+3$ imply that ${g^{\prime}}_{j^{\prime}} \in \mathfrak{M}$ and hence $\left.\phi_{4 k+3} \in \mathbb{M}\right]$, (2) there is an $i^{\prime} \geqq k$, such that $G_{1}, \cdots, G_{i^{\prime}}$ are in $\mathfrak{H}\left(\Phi_{1}, \cdots, \Phi_{4 k+3}\right)$.

Step IV. We construct $\phi_{4 k+4}, \Phi_{4 k+4}, k=0,1, \cdots$. This step is similar to Step III, with however $h$ instead of $G$, and $H$ instead of $g$. The concluding remarks are (1) $\Phi_{4 k+4} \in \mathfrak{M}^{P},(2)$ there is an $i^{\prime} \geqq k$, such that $h_{1}, \cdots, h_{i^{\prime}}$ are in $\mathfrak{A}\left(\phi_{1}, \cdots, \phi_{4 k+4}\right)$.

Steps I, II, III, and IV applied in rotation construct a biorthogonal series $\phi_{1}, \phi_{2}, \cdots, \Phi_{1}, \Phi_{2}, \cdots$. This is the desired construction.

Proof of the lemma. (a) is a consequence of remark (1) in Steps I and III, (b) is a consequence of remark (1) in Steps II and IV, (c), (d), (e) and (f) are the results of remark (2) in Steps I, II, IV and III respectively.

We can now dispense with the elements $g_{i}, G_{i}, h_{i}$, and $H_{i}$ which served to construct $\phi_{1}, \phi_{2}, \cdots, \Phi_{1}, \Phi_{2}, \cdots$.

LEMMA 19. If $\mathfrak{M}$ and $\mathfrak{M}^{P}$ are infinite-dimensional, there exists a biorthogonal series $\phi_{1}, \phi_{2}, \cdots, \Phi_{1}, \Phi_{2}, \cdots$ with the properties (1) $\left[\mathfrak{U}\left(\phi_{1}, \phi_{3}, \phi_{5}, \cdots\right)\right]=\mathfrak{M}$, (2) $\left[\mathfrak{U}\left(\Phi_{2}, \Phi_{4}, \Phi_{6}, \cdots\right)\right]=\mathfrak{M}^{P}$, (3) $\left[\mathfrak{U}\left(\phi_{1}, \phi_{2}, \cdots\right)\right]=\mathfrak{B}$, and (4) $\left[\mathfrak{A}\left(\Phi_{1}, \Phi_{2}\right.\right.$, $\cdots)] \doteq \mathfrak{B}^{*}$.

Proof. (1) Lemma $18\left(\right.$ a) implies that $\left[\mathfrak{A}\left(\phi_{1}, \phi_{3}, \phi_{5}, \cdots\right)\right] \subset \mathfrak{M}$. On the other hand, Lemma $18(\mathrm{c})$ shows that $\mathfrak{A}\left(\phi_{1}, \phi_{3}, \phi_{5}, \cdots\right) \supset\left\{g_{i}\right\}$. This and Lemma $16(\mathrm{a})$ show that $\left[\mathfrak{A}\left(\phi_{1}, . \phi_{3}, \phi_{5}, \cdots\right)\right] \supset \mathfrak{M}$. Thus we have $\mathfrak{M}$ $=\left[\mathfrak{A}\left(\phi_{1}, \phi_{3}, \phi_{5}, \cdots\right)\right]$.

(2) This follows in a similar fashion from Lemmas 18(b), (d) and 16(d).

(3) This follows from Lemmas 18(c), (e) and 16(c).

(4) Lemmas 18(d), (f) and 16(b) imply (4).

For completeness, we include the following lemma.

LEMMA 20. If $\mathfrak{M} \neq(0)$ is of dimension $n<\infty$, then there exists a biorthogonal series $\phi_{1}, \cdots, \phi_{n}, \Phi_{1}, \cdots, \Phi_{n}$, such that $\mathfrak{A}\left(\phi_{1}, \cdots, \phi_{n}\right)=\mathfrak{M}$.

Proof. We have $n \neq 0$. Suppose we have constructed $\phi_{1}, \cdots, \phi_{k}$, $\Phi_{1}, \cdots, \Phi_{k}$ for a $k<n$ with $\mathfrak{A}\left(\phi_{1}, \cdots, \phi_{k}\right) \subset \mathfrak{M}$. We wish to construct $\phi_{k+1}$, $\Phi_{k+1}$.

To do this we first show that $\mathfrak{A}\left(\phi_{1}, \cdots, \phi_{k}\right)$ is closed. (We may mention that in the case $k=0, \mathfrak{A}$ is empty and hence closed.) Suppose $h$ is a limit point 
of $\mathfrak{X}\left(\phi_{1}, \cdots, \phi_{k}\right)$ and $\left\{h_{i}\right\}$ is a sequence from $\mathfrak{A}\left(\phi_{1}, \cdots, \phi_{k}\right)$ such that $h_{i} \rightarrow h$. We have $h_{i}=\sum_{j-1}^{k} a_{i, j} \phi_{j}$ since $h_{i} \in \mathfrak{A}\left(\phi_{1}, \cdots, \phi_{k}\right)$. By Lemma 12, $a_{i, j}=\Phi_{j}\left(h_{i}\right)$. Since $\Phi_{j}$ is continuous and $h_{i} \rightarrow h$ we have that $a_{i, j}$ converges to an $a_{j}$, for $j=1, \cdots, \dot{k}$, as $i \rightarrow \infty$. Consequently $\sum_{j=1}^{k} a_{i, j} \phi_{j}$ converges to $\sum_{j=1}^{k} a_{j} \phi_{j}$ since $k$ is fixed. Thus $h=\lim \sum_{j-1}^{k} a_{i, j} \phi_{j}=\sum_{j-1}^{k} a_{j} \phi_{j}$ and hence $h \in \mathfrak{A}\left(\phi_{1}, \cdots, \phi_{k}\right)$. We have shown that $\mathfrak{A}\left(\phi_{1}, \cdots, \phi_{k}\right)$ contains all its limit points and hence is closed.

Since $k<n, \mathfrak{A}\left(\phi_{1}, \cdots, \phi_{k}\right)$ is included in but cannot equal $\mathfrak{M}$. Let $\phi_{k+1}$ be an element of $\mathfrak{M}$ not in $\mathscr{A}\left(\phi_{1}, \cdots, \phi_{k}\right)$. By the lemma of [B, chap. IV, $\S 3$, p. 57], there is a $\Phi_{k+1} \in \mathscr{B}^{*}$ such that $\Phi_{k+1}(h)=0$ for $h \in \mathfrak{A}\left(\phi_{1}, \cdots, \phi_{k}\right)$ and $\Phi_{k+1}\left(\phi_{k+1}\right)=1$. It is clear that $\phi_{k+1}, \Phi_{k+1}$ augment the series $\phi_{1}, \cdots, \phi_{k}$, $\Phi_{1}, \cdots, \Phi_{k}$.

We can in this manner construct $\phi_{1}, \cdots, \phi_{n}, \Phi_{1}, \cdots, \Phi_{n}$, with $\mathfrak{A}\left(\phi_{1}, \cdots, \phi_{n}\right) \subset \mathfrak{M}$. $\mathfrak{U}\left(\phi_{1}, \cdots, \phi_{n}\right)=\mathfrak{M}$ since $\mathfrak{M}$ is $n$-dimensional and $\mathfrak{A}\left(\phi_{1}, \cdots, \phi_{n}\right)$ is closed.

8. ThEOREM. If $\mathfrak{B}$ is a reflexive separable Banach space with a separable conjugate space $\mathfrak{B}^{*}$, and if $\mathfrak{M}$ is a non-empty additive closed subset of $\mathfrak{B}$, then there exists a projection whose range is $\mathfrak{M}$. (In $\$ 4$, a projection is defined as a c.a.d.d.transformation $E$ with $E^{2}=E$.)

Proof. If $\mathfrak{M}=(0)$, let $E=0$.

If $\mathfrak{M}$ is finite-dimensional and not equal to (0) we consider the orthogonal set, $\phi_{1}, \cdots, \phi_{n}, \Phi_{1}, \cdots, \Phi_{n}$, with $\mathfrak{A}\left(\phi_{1}, \cdots, \phi_{n}\right)=\mathfrak{M}$ given by Lemma 20 . We then take the $E$ defined in Lemma 14 whose range is $\mathfrak{A}\left(\phi_{1}, \cdots, \phi_{n}\right)=\mathfrak{M}$.

If $\mathfrak{M}^{P}$ is finite-dimensional, we apply one of the preceding results to obtain an $E^{0}$ which projects $\mathfrak{B}^{*}$ on $\mathfrak{M}^{P}$. By Lemmas 9 (c) and $1(\mathrm{c})$, the set of zeros of $E^{0 *}$ is $\left(\mathfrak{M}^{P}\right)^{P}=\mathfrak{M}$. $E^{0 *}$ is a projection by Lemma 11 . Applying Lemma 11 again, we obtain that $E=1-E^{0 *}$ is a projection with range $\mathfrak{M}$.

Thus we may suppose that both $\mathfrak{M}$ and $\mathfrak{M}^{P}$ are infinite-dimensional. Consequently we may apply Lemma 19, and obtain the biorthogonal series $\phi_{1}, \phi_{2}, \cdots, \Phi_{1}, \Phi_{2}, \cdots$ of that lemma.

We define an additive transformation $E^{\prime}$ with domain $\mathfrak{A}\left(\phi_{1}, \phi_{2}, \cdots\right)$ as follows. If $h \in \mathcal{A}\left(\phi_{1}, \phi_{2}, \cdots\right)$ then for all sufficiently large $k$, we may express $h$ in the form $\sum_{i=1}^{2 k} a_{i} \phi_{i}$, using zero coefficients when necessary. Let $E^{\prime} h=\sum_{j-1}^{k} a_{2 j-1} \phi_{2 j-1}$, that is, we suppress in the expansion for $h$ the $\phi_{i}$ 's with even subscripts. Lemma 12 shows that $E^{\prime} h$ is the same no matter which permissible $k$ is chosen. Hence the transformation $E^{\prime}$ is uniquely defined. It is clearly additive.

Lemma 19 (c) shows that $\mathfrak{A}\left(\phi_{1}, \phi_{2}, \cdots\right)$, the domain of $E^{\prime}$, is dense. This and Lemma 7 imply that $E^{\prime *}$ exists and is closed and additive. Consider $\Phi_{2 s-1}$ for a given $s=1,2, \cdots$. Let $h=\sum_{i=1}^{2 k} a_{i} \phi_{i}$ and we may suppose $k>s$. Then since $\Phi_{s}\left(\phi_{j}\right)=\delta_{s, j}$, 


$$
\begin{aligned}
\Phi_{2 s-1}\left(E^{\prime} h\right) & =\Phi_{2 s-1}\left(\sum_{j=1}^{k} a_{2 j-1} \phi_{2 j-1}\right)=a_{2 s-1} \\
& =\Phi_{2 s-1}\left(\sum_{i=1}^{2 k} a_{i} \phi_{i}\right)=\Phi_{2 s-1}(h) .
\end{aligned}
$$

Also

$$
\Phi_{2 \triangleleft}\left(E^{\prime} h\right)=\Phi_{2 \triangleleft}\left(\sum_{j=1}^{k} a_{2 j-1} \phi_{2 j-1}\right)=0=0(h) .
$$

These equations for all $h \in \mathcal{A}\left(\phi_{1}, \phi_{2}, \cdots\right)$ yield by $(\beta)$ of $\S 3$ that $E^{\prime *} \Phi_{2 s-1}$ exists and equals $\Phi_{2 \varepsilon-1}$ and $E^{\prime *} \Phi_{2 \varepsilon}$ exists and equals 0 . Since $E^{\prime *}$ is additive, we have then that its domain, $\mathfrak{D}^{*}$, contains $\mathscr{Y}\left(\Phi_{1}, \Phi_{2}, \cdots\right)$.

Since $\mathscr{H}\left(\Phi_{1}, \Phi_{2}, \cdots\right)$ is dense, $\mathfrak{D}^{*}$ is also dense. Thus $E^{\prime}$ is a transformation whose adjoint has a dense domain. Lemma 8 now shows that $\left[E^{\prime}\right]$ exists. Let $E=\left[E^{\prime}\right]$. The corollary to Lemma 8 shows that $E^{*}=E^{\prime *}$. Since the domain of $E^{\prime}$ is dense, so also is the domain of $E$ which includes it. Since $E^{\prime}$ is additive, $E$ is also. Thus $E$ is c.a.d.d.

We next show that the range of $E, \mathfrak{R}$, is $\mathfrak{M}$. Let $\mathfrak{M}_{1}$ denote the $h$ 's $\in \mathfrak{B}$ such that $E h=h . E-1$ is closed by Lemma 9 (a) and consequently Lemma 9(b) shows that $\mathfrak{M}_{1}$, the set of zeros of $E-1$, is also closed. Now $E \phi_{2 s-1}$ $=E^{\prime} \phi_{2 s-1}=\phi_{2 s-1}$ and hence $\mathfrak{M}_{1}$ contains $\phi_{2 s-1}$. Since $\mathfrak{M}_{1}$ is closed and additive, $\mathfrak{M}_{1}$ must contain $\left[\mathfrak{H}\left(\phi_{1}, \phi_{3}, \cdots\right)\right]$. Lemma $19\left(\right.$ a) states that $\left[\mathscr{A}\left(\phi_{1}, \phi_{3}, \cdots\right)\right]$ $=\mathfrak{M}$ and thus $\mathfrak{M}_{1} \supset \mathfrak{M}$. Clearly $\mathfrak{R} \supset \mathfrak{M}_{1}$ and hence $\mathfrak{R} \supset \mathfrak{M}$.

On the other hand, consider $\mathfrak{R}^{*}$ the set of zeros of $E^{*}=E^{\prime *}$. Lemma 9 (b) shows that $\mathfrak{R}^{*}$ is closed. We also have from the above that $E^{*} \Phi_{2 s}=E^{\prime *} \Phi_{2 s}=0$. This means that $\Phi_{2 s} \in \mathfrak{R}^{*}$ and since $\mathfrak{R}^{*}$ is closed and additive we have that $\mathfrak{R}^{*} \supset\left[\mathfrak{H}\left(\Phi_{2}, \Phi_{4}, \cdots\right)\right]$. Lemma $19(\mathrm{~b})$ states that $\mathfrak{M}^{P}=\left[\mathfrak{H}\left(\Phi_{2}, \Phi_{4}, \cdots\right)\right]$, and thus $\mathfrak{R}^{*} \supset \mathfrak{M}^{P}$. Taking the orthogonal complements, we obtain $\mathfrak{R}^{* P} \subset \mathfrak{M}$. Lemma 9 (c) shows that $[\Re]=\Re^{* P}$ and thus $\Re \subset[\Re]=\mathfrak{N}^{* P} \subset \mathfrak{R}$. (This also follows from the fact that the range of $E^{\prime}$ is included in $\mathfrak{M}$ and $E$ is the closure of $E^{\prime}$.) With the result of the previous paragraph, this yields $\Re=\mathfrak{M}$. The previous paragraph also yields $\mathfrak{R} \supset \mathfrak{M}_{1} \supset \mathfrak{M}$. This now implies $\mathfrak{M}_{1}=\mathfrak{M}=\mathfrak{R}$.

Since $\Re=\mathfrak{M}_{1}$, we have that for $h \in \Re, E h=h$. Thus for every $f$ for which $E f$ is defined, we have $E(E f)=E f$. This is equivalent to $E^{2}=E$.

We have shown that $E$ is a c.a.d.d. transformation with $E^{2}=E$ and that the range of $E$ is $\mathfrak{M}$. This completes the proof of the theorem.

If we consider Lemma 10(a), we obtain the following corollary.

CoROLLARY. Under the hypotheses of the above theorem, $\mathfrak{M}$ has a quasi-complement.

9. In this section, we show that a quasi-complement, which is not a complement, that is, for which $\mathfrak{M} \cdot+\cdot \mathfrak{N}$ is not $\mathfrak{B}$, can always be increased or decreased in such a way that the result is still a quasi-complement. 
Lemma 21. Suppose $\mathfrak{R}$ is a closed additive set and $f$ is an element not in $\mathfrak{R}$. Then the set $\{a f\} \cdot+\cdot \mathfrak{N}$ is closed.

Proof. Let $d=$ gr.l.b. $(|f+g|, g \in \mathfrak{N})$. Since $f$ is not in $\mathfrak{N}, d \neq 0$. We also have $|a| \cdot d \leqq|a f+g|$ for every $g \in \mathfrak{R}$ and every $a$.

Let $h$ be a limit point of $\{a f\} \cdot+\cdot \mathfrak{R}$ and $a_{n} f+g_{n}$ a sequence which approaches $h$. This sequence is convergent and from the above we have

$$
\begin{aligned}
d \cdot\left|a_{n}-a_{m}\right| & \leqq\left|\left(a_{n}-a_{m}\right) f+g_{n}-g_{m}\right| \\
& =\left|a_{n} f+g_{n}-\left(a_{m} f+g_{m}\right)\right| \rightarrow 0 .
\end{aligned}
$$

Thus the $a_{n}$ 's converge and consequently the $g_{n}$ 's do also. Let the limits of these be respectively $a$ and $g$. It follows that $h=\lim \left(a_{n} f+g_{n}\right)=a f+g \in\{a f\}$ $\cdot+\cdot \mathfrak{N}$ and this last set is closed.

TheOREM. Let $\mathfrak{M}$ and $\mathfrak{R}$ be quasi-complements but not complements. Then there is an $\mathfrak{N}^{\prime} \supset \mathfrak{N}$ but $\neq \mathfrak{N}$ which is also a quasi-complement to $\mathfrak{M}$. There is also an $\mathfrak{R}^{\prime \prime} \subset \mathfrak{N}$ but $\neq \mathfrak{N}$ which is a quasi-complement to $\mathfrak{M}$.

Proof. Since $\mathfrak{M}$ and $\mathfrak{R}$ are not complements there is an $f$ not in $\mathfrak{M} \cdot+\cdot \mathfrak{R}$. $f$ is not in $\mathfrak{N}$ and thus $\mathfrak{N}^{\prime}=\{a f\} \cdot+\cdot \mathfrak{N}$ is closed. We show that $\mathfrak{M}$ and $\mathfrak{N}^{\prime}$ are quasi-complements.

Suppose $h \in \mathfrak{M} \cdot \mathfrak{N}^{\prime}$. Then $h \in \mathfrak{M}$ and $h=a f+g$ for a $g \in \mathfrak{N}$. Now if $a \neq 0$, we have $f=(1 / a) h+(-1 / a) g \in \mathfrak{M} \cdot+\cdot \mathfrak{N}$ contrary to our hypothesis on $f$. Hence $a=0$ and $h=g \in \mathfrak{M} \cdot \mathfrak{R}=(0)$. We have then $h=0$ and $\mathfrak{M} \cdot \mathfrak{N}^{\prime}=(0)$. We also have $\mathfrak{M} \cdot+\cdot \mathfrak{N}^{\prime} \supset \mathfrak{M} \cdot+\cdot \mathfrak{N}$ and the latter is dense. Hence $\mathfrak{M} \cdot+\cdot \mathfrak{N}^{\prime}$ is also dense. We have now shown that $\mathfrak{M}$ and $\mathfrak{N}^{\prime}$ are quasi-complements.

We next show the existence of $\mathfrak{N}^{\prime \prime}$, a proper subset of $\mathfrak{N}$, which is also a quasi-complement of $\mathfrak{M}$. Consider $\mathfrak{M}^{P}$ and $\mathfrak{R}^{P}$. By the above, we can take a set $\mathfrak{R}^{P^{\prime}}$, which properly includes $\mathfrak{N}^{P}$ and which is also a complement to $\mathfrak{M}^{P}$. The corollary to Lemma 11 shows that $\mathfrak{M}$ and $\mathfrak{N}^{\prime \prime}=\left(\mathfrak{N}^{P^{\prime}}\right)^{P}$ are quasi-complements. Since $\mathfrak{N}^{P} \subset \mathfrak{N}^{P^{\prime}}$, we have $\mathfrak{N} \mathfrak{N}^{\prime \prime}$. Furthermore, we cannot have $\mathfrak{N}=\mathfrak{N}^{\prime \prime}$ for then $\mathfrak{N}^{P}=\mathfrak{N}^{\prime \prime P}=\left(\mathfrak{N}^{P \prime}\right)^{P P}=\mathfrak{N}^{P^{\prime}}$ contrary to our choice of $\mathfrak{N}^{P^{\prime}}$ as not equal to $\mathfrak{R}^{P}$. Thus $\mathfrak{N}^{\prime \prime}$ is a proper subset of $\mathfrak{N}$, which is a quasi-complement for $\mathfrak{M}$.

CoROLlaRY. If $E$ is an unbounded projection on $(\mathfrak{M})$, it may be extended to an $E^{\prime}$ which is also a projection on $\mathfrak{M}$ or contracted to an $E^{\prime \prime}$ which is also a projection on $\mathfrak{M}$.

Proof. Let $\mathfrak{R}$ be the set of zeros of $E$. By Lemma $10(a), \mathfrak{M}$ and $\mathfrak{R}$ are quasi-complements. By the above theorem we may increase $\mathfrak{N}$ to an $\mathfrak{N}^{\prime}$ which is still a quasi-complement to $\mathfrak{M}$. By Lemma $10(\mathrm{~b})$, we have a projection $E^{\prime}$ with range $\mathfrak{M}$ and zeros $\mathfrak{N}^{\prime}$. This is an extension of $E$ with which it agrees on $\mathfrak{M} \cdot+\cdot \mathfrak{R}$.

Similarly $\mathfrak{R}^{\prime \prime}$ may be used to find a contraction of $E$. 


\section{BIBLIOGRAPHY}

[B] S. Banach, Theorie des operationes linéaires, Warsaw, 1932.

[BS] H. F. Bohnenblust and A. Sobczyk, Bull. Amer. Math. Soc. vol. 44 (1938) pp. 91-93.

[KS] M. Krein and V. Smulian, Ann. of Math. (2) vol. 41 (1940) pp. 556-583, in particular chap. 2.

[M1] F. J. Murray, Trans. Amer. Math. Soc. vol. 39 (1936) pp. 83-100.

[M2] - Trans. Amer. Math. Soc. vol. 41 (1937) pp. 138-152.

[M3] Linear transformations in Hilbert space, Princeton, 1941.

[M4] - Bull. Amer. Math. Soc. vol. 48 (1942) pp. 76-93.

[v.N] J. von Neumann, Ann. of Math. (2) vol. 33 (1932) pp. 294-310.

[S] A. Sobczyk, Duke Math. J. vol. 8 (1941) pp. 78-106.

\section{Columbia University,}

NEW YORK, N. Y. 